\title{
Magnetic Resonance Imaging of Skeletal Metastatic Sites with Contrast
}

National Cancer Institute

\section{Source}

National Cancer Institute. Magnetic Resonance Imaging of Skeletal Metastatic Sites with

Contrast. NCI Thesaurus. Code C137932.

Magnetic resonance imaging of skeletal metastatic sites with the use of a contrast agent to enhance the image. 